\title{
Valuing knowledge of the cost and cost-effectiveness of medicines
}

\section{Commentary on ... A Awareness of the cost of psychotropic medication among doctors ${ }^{\dagger}$}

\author{
Sarah Byford, ${ }^{1}$ Iris Molosankwe ${ }^{1}$
}

The Psychiatrist (2010), 34, 369-370, doi: 10.1192/pb.bp.110.030155

${ }^{1}$ King's College London

Correspondence to Sarah Byford (s.byford@iop.kcl.ac.uk)

\begin{abstract}
Summary Knowledge of the cost of pharmaceuticals is an important step towards cost-effective prescribing, yet evidence presented by Singh and colleagues highlights a lack of awareness of the cost of psychotropic medication among doctors in one NHS foundation trust and failures in the dissemination of cost data. These findings support the existence of substantial barriers to the success of cost-effective prescribing strategies in the UK. The next, and more challenging step, is to explore knowledge of the relative cost-effectiveness of pharmaceuticals, since knowledge of cost alone is inadequate to ensure prescribing practices make a meaningful constribution to the efficient use of scarce health service resources.
\end{abstract}

Declaration of interest None.
Prescription costs account for a significant proportion of the National Health Service (NHS) budget, with evidence showing a steady rise over the years. In 2008, NHS expenditure on medicines in England was estimated at $£ 11.6$ billion, with hospital use accounting for $£ 3.3$ billion of this total. ${ }^{1}$ This substantial drugs bill has prompted the Department of Health to explore areas in which they can make savings without compromising the quality of patient care. ${ }^{2}$ Common strategies include 'generic substitution', in which cheaper generic drugs are substituted for equivalent but more expensive brand name medications, and 'therapeutic substitution', where lower-cost treatments within a particular class of drugs are substituted for higher-cost alternatives, where equivalence is known or assumed. ${ }^{3}$ To be successful, such strategies require knowledge of the cost of medications on the part of those with responsibility for prescribing.

\section{Knowledge of the prices of medicines}

In a service evaluation of the awareness of the cost of psychotropic medication among doctors in a London NHS foundation trust, Singh and colleagues report a poor level of knowledge, with only $5 \%$ of doctors surveyed claiming to know the price of the medications they prescribe most frequently. ${ }^{4}$ Thus, despite Department of Health policies to encourage lower-cost prescribing, the crucial ingredient for

†See original paper, pp. 364-366 and commentary, pp. 367-368, this issue. success - knowledge - appears to be lacking, at least in the hospital sector.

This important finding raises a number of questions about the role and responsibilities of clinicians in the efficient allocation of scarce health service resources. The authors suggest a number of explanations for their findings including inadequate training, information overload and differences in perspective towards, or a lack of concern for, issues of cost-effective prescribing. Previous commentators have highlighted inertia in prescribing behaviour and a lack of willingness to take cost into consideration, even when good evidence of cost-effectiveness exists. ${ }^{5}$ A further concern is the perceived conflict between the role of keeper of the public purse and the basic principles of medical ethics, particularly beneficence and autonomy, which focus on the individual, as opposed to the principle of justice, which sits more comfortably with economic theory. $^{6}$

However, it is not just the clinicians we should be looking to for answers, but also the ability of policy, governing and academic bodies to successfully disseminate accessible cost and cost-effectiveness messages to those who make resource allocation decisions on a daily basis, and indeed to adequately explain the importance of such messages. The work by Singh and colleagues suggests failures in dissemination, with only $35 \%$ of the doctors surveyed stating that they had easy access within their hospitals to information about the cost of the drugs they prescribe and only $34 \%$ being aware of a system within the trust to promote cost-effective prescribing. 


\section{Cost v. cost-effectiveness of medicines}

There is one limitation to the work presented by Singh and colleagues, which merits closer consideration, and this relates to the distinction between 'cost' and 'costeffectiveness'. The authors set out to assess six standards relating to cost-effective prescribing. However, we would argue that these standards, although overlapping with issues of cost-effectiveness, focus more on the cost of prescribing than the cost-effectiveness. Cost-effective prescribing does not necessarily require knowledge of the exact cost of a drug; instead, it requires evidence from economic evaluations which consider cost data alongside evidence of effectiveness.

Economic evaluation involves the systematic attempt to identify, measure and compare all relevant costs and outcomes of alternative resource allocation decisions in order to ensure that scarce healthcare resources are allocated in a way that produces the greatest benefit for patients. ${ }^{7}$ An efficient change in resource allocation is one which generates greater patient benefit for the same level of expenditure, or produces the same level of patient benefit for lower cost. Thus, although it is true that prescribing generically rather than by trade name is a cost-effective (or efficient) strategy, this is not just because the generic drug is cheaper but also because the effectiveness of the two drugs is known to be equal. However, selecting the cheapest drug within a particular class of medicines where those medicines are known to vary in their effectiveness and side-effect profile will not guarantee a cost-effective improvement. In fact, it is entirely possible for a more expensive drug to be more cost-effective than a cheaper alternative if it generates additional patient benefits that are valued highly enough to justify the extra expense and/or if it produces savings elsewhere in the health or wider social care system by reducing the patient's need for other services. $^{8}$

\section{Accessibility of cost-effectiveness evidence}

So, to ensure cost-effective prescribing, clinicians must be familiar with evidence from economic evaluations, not just the cost of the pharmaceuticals they are prescribing. This requirement adds a substantial burden to healthcare professionals, who cannot possibly be expected to read, critique and digest every economic evaluation related to all of the drugs they prescribe. Instead, policy makers around the world have been developing systems to synthesise and disseminate evidence of cost-effectiveness. In England and Wales, this is the task of the National Institute for Health and Clinical Excellence (NICE), charged with providing national guidance on the promotion of good health and the prevention and treatment of ill health. ${ }^{9}$ In making its recommendations, NICE is required to consider evidence of both effectiveness and cost-effectiveness. ${ }^{10}$

Although such guidance is intended to support treatment decisions in the NHS, much evidence exists to suggest that prescribing patterns in routine practice often fall short of guideline standards, including prescribing of psychotropic medications. ${ }^{11}$ The impact of evidence from NICE and other similar organisations is dependent not only upon the availability of synthesised evidence, but also the accessibility of this evidence and indeed the attitudes of prescribing clinicians. As Towse \& Wells highlighted some 15 years ago, cost-effective prescribing 'requires decision makers to believe that such information is relevant'. ${ }^{12}$ The study by Singh and colleagues clearly illustrates that the interpretation of notions of cost-effectiveness can be confusing and suggests that there is still much to do to change the attitudes and understanding of those with responsibility for prescribing.

More emphasis on the value of knowledge of both the cost and cost-effectiveness of medicines is needed to ensure the future success of strategies to promote cost-effective prescribing. This can be achieved through clinical training and improvements to existing dissemination practices, with particular attention to the production of evidence that is easily accessible.

\section{About the authors}

Sarah Byford, Reader, Centre for the Economics of Mental Health, Iris Molosankwe, Research Worker, King's College London.

\section{References}

1 NHS Information Centre. Hospital Prescribing, 2008: England. Health and Social Care Information Centre, 2009 (http://www.ic.nhs.uk/webfiles/ publications/Primary\%20Care/Prescriptions/hospre08/ Hospital_prescribing_2008_report2.pdf).

2 Pearson C. Strategies to Achieve Cost-Effective Prescribing. Interim Guidance for Primary Care Trusts. Department of Health, 2007.

3 Polinski JM, Maclure M, Marshall B, Cassels A, Agnew-Blais J, Patrick $A R$, et al. Does knowledge of medication prices predict physicians' support for cost effective prescribing policies? Can J Clin Pharmacol 2008; 15: e286-94.

4 Singh DK, Khawaja S, Pala I, Khaja J, Krishnanu R, Walker $H$, et al. Awareness of the cost of psychotropic medication among doctors: a service evaluation. Psychiatrist 2010; 34: 364-366.

5 Bloor K, Freemantle N. Promoting Cost-Effective Prescribing in the UK National Health Service. Centre for Health Economics, University of York, 1997.

6 Byford S, Barrett B. Ethics and economics: the case for mental health care. Adv Psychiatr Treat 2010; in press.

7 Drummond MF, Sculpher MJ, Torrance GW, O'Brien BJ, Stoddart GL. Methods for the Economic Evaluation of Health Care Programmes. Oxford University Press, 2005.

8 Freemantle N, Henry D, Maynard A, Torrance G. Promoting costeffective prescribing. BMJ 1995; 310: 955-6.

9 National Institute for Health and Clinical Excellence. A Guide to NICE. NICE, 2005.

10 National Institute for Health and Clinical Excellence. Guide to the Methods of Technology Appraisal. NICE, 2008.

11 Moran M, Raju B, Saunders J, Meagher D. Achieving evidence-based prescribing practice in an adult community mental health service. Psychiatr Bull 2006; 30: 51-5.

12 Towse A, Wells N. Promoting cost effective prescribing. BMJ 1995; 311 126 